\title{
Kerr Gated Resonance Raman Spectroscopy In Light Fastness Studies Of Ink Jet Prints
}

Katri Vikman ${ }^{1 *}$, Hanna Ittil ${ }^{1}$, Pavel Matousek ${ }^{2}$, Michael Towrie ${ }^{2}$, Anthony W. Parker ${ }^{2}$ and Tapani Vuorinen ${ }^{1}$

${ }^{1}$ Helsinki University of Technology, Laboratory of Forest Products Chemistry, Vuorimiehentie 1, FIN-02150 Espoo, Finland

${ }^{2}$ Central Laser Facility, CCLRC Rutherford Appleton Laboratory, Chilton, Didcot, Oxfordshire OX11 0QX, United Kingdom

*Corresponding author. Tel. +358-9-4514253, fax. +358-9-4514259, e-mail: katri.vikman@hut.fi.

\begin{abstract}
Resonance Raman spectroscopy offers several advantages in the studies of ink jet prints stemming from its inherent selectivity and sensitivity. These include the possibility to obtain direct information on the chemical interactions between the paper and ink that play a major role in long-term print fastness. However, resonance Raman techniques can suffer from obtrusive fluorescence from colorants or printing substrate and this severely complicates the Raman measurements.

The aim of this investigation was to evaluate the applicability of resonance Raman spectroscopy combined with a Kerr gate fluorescence rejection system to gain information on the light fastness of ink jet prints. This was studied using a set of experimental coatings and inks with known compositions. The results indicate that Kerr gated resonance Raman spectroscopy is applicable to the light fastness studies of ink jet prints owing to the effective suppression of the sample-related fluorescence. Moreover, detailed molecular information can be obtained by tuning the laser to get selective resonance enhancement. This enables specific analyses of chemical paper-ink interactions, which generally contribute decisively to the light fastness of ink jet prints.
\end{abstract}

KEYWORDS: Ink jet, resonance Raman spectroscopy, Kerr gate, light fastness 


\section{INTRODUCTION}

Ink jet printing is one of the major printing technologies because of its versatility. During recent years its use has become common particularly in small-scale color printing and in lowcost variable data printing applications. ${ }^{1,2}$ Manifold criteria are generally set for the ink jet print quality, eligible features typically including large color gamut, sharp detail rendering, and long-term fastness of the printed image towards i.e. light and moisture. These characteristics are highly dependent on the properties of both papers and inks and their chemical and physical interactions. ${ }^{3-5}$ Traditional optical print quality measurement methods do not yield enough information to deduce the nature of the chemical interactions between the paper and the ink prevailing for ink jet printing. Consequently, other analytical methods are needed. Vibrational spectroscopy, or IR and Raman spectroscopy, offers several advantages in print studies over many other analytical techniques. The most remarkable benefit is the possibility to investigate the prints regardless of their physical state, and thereby obtain direct information about the actual paper-ink-interactions. In other words, both the inks and papers can be examined without destructive and often tedious sample preparation. Another advantageous feature is the opportunity to accomplish both surface and depth profiling measurements.

Earlier studies have shown that Raman spectroscopic methods are applicable to the analyses of ink jet prints. ${ }^{6-9}$ As the method is sensitive particularly to the aromatic structures and double bonds, it enables the detection of even minor amounts of the colorants on the paper surfaces and analysis of ageing, e.g. light-induced changes. Furthermore, resonance Raman spectroscopy offers additional advantages owing to its molecular specificity and high sensitivity. ${ }^{10}$ With this method, certain chemical structures, i.e. chromophores of colorant molecules, can be selectively enhanced using an appropriate laser wavelength tuned to match an electronic absorption band of the sample of interest. ${ }^{11}$ However, despite the many benefits that there are to ink jet print studies, resonance Raman spectroscopy often suffers from obtrusive fluorescence, originating for example from various ink jet colorants and paper coating components. ${ }^{7-9}$ This behavior complicates greatly the measurements and frequently makes resonance Raman impracticable or severely hampers spectral interpretation. Several solutions to eliminating the fluorescence problem have been suggested. In analyses of colorants and ink jet inks, the use of near-infrared excitation (NIR-FTR) and surfaceenhanced resonance Raman scattering (SERRS) has been one strategy as a means to avoid or attenuate the sample-related fluorescence. ${ }^{6,11}$ Nevertheless according to the results, fluorescence still proved to be a problem in NIR-FTR measurements, whereas in SERRS, the required intrusive sample pre-treatment prevented the prints from being studied naturally. The 
fluorescence problem has also been alleviated to some extent by using an immersion objective in the NIR-Raman measurements of the prints, or by collecting the spectra using laser wavelengths in the UV region. ${ }^{7,12}$.

One of the best solutions for fluorescence rejection is the temporal gating of the detected signal and several groups have worked in this area. ${ }^{13-20}$ This approach utilizes the difference between the temporal characteristics of Raman and fluorescence signals. For example; as the sample is excited using a high repetition rate picosecond laser beam, an instantaneous Raman signal is scattered first, whereas the longer-lived fluorescence is emitted on a substantially longer time-scale, typically tens of nanoseconds. Consequently, the fluorescence (or indeed phosphorescence) can be rejected in the time domain. This procedure has been implemented by using a Kerr gate technique ${ }^{18-20}$, in which the fluorescence rejection is based on a transient change in the optical properties of the gate material, e.g. carbon disulfide $\left(\mathrm{CS}_{2}\right)$.

Time-resolved Raman spectroscopy has been utilized in the analyses of both dyes and solid samples. For example, the electronic and vibrational relaxation of photoexcited transazobenzene in solution has been investigated ${ }^{21}$. The Kerr gate system has made possible resonance Raman spectroscopy of highly fluorescing dyes and even laser dyes Coumarin 480 and Rhodamine 6G. ${ }^{19,20}$ The applicability of Kerr gate picosecond time-resolved Raman spectroscopy to the studies of powders and films has also been explored ${ }^{22}$. The method notably reduced the fluorescent background originating from solid materials. Furthermore, the Kerr gate technique has been successfully applied to the fluorescence rejection of pulp sheets $^{23}$.

The objective of this study is to evaluate the applicability of resonance Raman spectroscopy equipped with an optical Kerr gate for investigating the light fastness of ink jet prints. The work has been performed on a set of experimental coatings and inks of known compositions.

\section{EXPERIMENTAL SECTION}

Sample preparation. A model coating set was generated, where the structure of the coating layer, absorption properties, and net-charge of the coating were altered by varying the pigment and polymer compositions (Table 1). The coating pigments were precipitated calcium carbonate (PCC, Jetcoat 30 from Specialty Minerals) and kaolin (Digitex from Engelhard). Both pigments were used as received: PCC as a slurry with $25 \%$ solids content, consisting of pigment and carboxymethyl cellulose (CMC) as a thickener, and the kaolin as a pigment powder. The absorption properties of the coatings were modified using different types of binder. These were polyvinyl alcohol (PVA, Celvol 107 from Air Products, degree of hydrolysis $98.0-98.8 \%$ ) and weakly cationic styrene acrylate latex (SA, Raiprint 300 from 
Raisio Chemicals, charge $236 \mu \mathrm{molkg}^{-1}$ at $\mathrm{pH}$ 8). According to the manufacturer, the latex was stabilized with a cationic starch. The net charge of the coatings was varied using different dispersants for the kaolin pigment. These were cationic polydiallyl dimethyl ammonium chloride (poly-DADMAC, Cartafix VXT from Clariant International Ltd., cationic charge density $2200 \mathrm{mEqkg}^{-1}$ ) and weakly cationic starch (Raisamyl 402 from Raisio Chemicals, DS $=0.018$, cationic charge density $100 \mathrm{mEqkg}^{-1}$ ). Moreover, $1.5 \mathrm{pph}$ of CMC (Finnfix 10 from Noviant, DS $=0.6-0.95$ ) was added to each coating color to increase its viscosity. The $\mathrm{pH}$ of the coating colors was not adjusted. The base paper was regular surface-sized copy paper ( 80 $\mathrm{gm}^{-2}$ ) and the target amount of coating was $10 \mathrm{gm}^{-2}$. The coating was made with a CLC laboratory blade coater at a $50^{\circ}$ blade angle and a coating speed of $350 \mathrm{~m} / \mathrm{min}$. The coated papers were uncalendered.

Three different model inks were used (Table 2), for which two soluble azo colorants with different chemical properties (CI Direct Yellow 86, DY86; CI Direct Violet 107, DV107) and one pigment dispersion (CI Pigment Yellow 83, PY83) were chosen. Detailed information on the molecular structure of DY86 and PY83 can be found elsewhere, i.e., from the Colour Index. Information on the molecular structure of DV107 is not publicly available. The dyebased colorants were purchased from Avecia Ltd as aqueous solutions, and the pigment dispersion from Clariant International Ltd. The latter contained 35\% of colorant (average particle size $40 \mathrm{~nm}$ ) and 15\% of 1,2-propylene glycol (PG), whereas the exact compositions of the purchased dye solutions are not known. The added co-solvents were diethylene glycol (DEG, purum; $\geq 98.0 \%$ GC) and 2-pyrrolidone (purity grade: purum; $\geq 99.0 \%$ GC), both from Sigma-Aldrich.

Solid single-color areas were printed on the model coatings with an Epson Stylus Photo 890 piezo ink jet printer using the model inks. The printed samples were exposed to artificial sunlight for 100 hours with a Suntest CPS+ xenon arc lamp, and the color difference value $\Delta \mathrm{E}^{*}$ based on CIEL*a*b* color coordinates was used as a measure of light fastness. The detailed description of the light fastness measurement procedure is given in an earlier paper ${ }^{8}$. The light fastness values after $100 \mathrm{~h}$ exposure for the DY86 DEG ink on various model coatings are listed in Table 1.

Kerr Gated Resonance Raman Spectroscopy. The Kerr gate set-up used in this study consisted of two crossed polarizers, and a Kerr medium $\left(\mathrm{CS}_{2}\right)$. Simultaneously with the Raman scattering from the sample, a short gating pulse (1 ps) creates a transient ( 4 ps) anisotropy within the Kerr medium that selectively rotates the polarization of the Raman light but the majority of the fluorescence that arrives later is unaffected. After rotation the Raman signal is transmitted through a cross-polarizer, but the fluorescence light is blocked. The laser 
equipment of the present measurement set-up provided $800 \mathrm{~nm}, 1 \mathrm{ps}, 2-3 \mathrm{~mJ}$ fundamental pulses at $1 \mathrm{kHz}$ repetition rate. The fundamental laser output was split in two such that one part was taken for the gating pulse to drive the Kerr gate, and the remaining part was used as a probe beam yielding at the sample $\sim 1 \mu \mathrm{J}$, i.e. $1 \mathrm{~mW}$. The probe beam was weakly focused down onto sample to around $0.3 \mathrm{~mm}$ diameter spot. The scattered Raman light was collected at $90^{\circ}$ angle with respect to the incident beam. Carbon disulfide $\left(\mathrm{CS}_{2}\right)$ was used as a Kerr gate material, and $470 \mathrm{~nm}$ was chosen as a probe wavelength. More detailed description of the equipment is given elsewhere. ${ }^{18-20,23}$

Prior to the measurements, the spectrometer was calibrated using a polyethylene terephtalate film. The printed samples were mounted on to the sample stage at $45^{\circ}$ angle to the probe beam, and rotated during the measurement in order to prevent burning. The liquid inks were measured in an $0.5 \mathrm{~mm}$ diameter ink stream in an open flow perpendicular to the probe beam. The acquisition period was $20 \mathrm{~s}$, and ten acquisitions for each gate - probe time delay were collected. Thus, the duration of one measurement cycle was $400 \mathrm{~s}$. Altogether five cycles were recorded for the liquid inks and DY86 DEG prints, whereas for DV107 and PY83 prints, only three cycles were collected.

Data Analysis. The spectral data were analyzed using the principal component analysis (PCA), which is a multivariate data analysis method. The mathematical background of PCA is described in detail elsewhere ${ }^{24}$. No data scaling or other transformations were performed to the spectral data prior to the principal component analysis to obtain more realistic impression on the possible spectral changes.

\section{RESULTS AND DISCUSSION}

Fluorescence suppression. Preliminary measurements of the resonance Raman spectra of printed samples were accomplished with $400 \mathrm{~nm}$ excitation wavelength, for all the studied colorants absorb light strongly at this wavelength. However, despite the use of Kerr gate, the resulting spectra were found to contain an extremely high amount of fluorescence. This can be attributed to the optical brightener included in the base paper. Consequently, as the absorption region of the studied colorants in the visible range extends to the higher wavelengths the following experiments were performed using the excitation wavelength 470 $\mathrm{nm}$ to exclude the contribution from the optical brightener. Figure 1 illustrates the effect of Kerr gate rejection on the quality of the resonance Raman spectra of yellow dye-based ink jet prints at $470 \mathrm{~nm}$ excitation wavelength. The uppermost curve is the resonance Raman spectrum of an unexposed ink jet print measured without the Kerr gate. It is evident that the strong background fluorescence almost completely obscures the Raman bands originating 
from the sample. In contrast, in the corresponding spectrum measured with the Kerr gate (the second curve), the Raman bands are clearly visible. Thus, the Kerr gate system effectively suppresses the fluorescence originating from the sample. The resonance Raman spectrum of the unprinted coating containing PCC as a sole coating pigment (P100:0) is shown in Figure 1 for control. Kaolin used in this study does not give rise to any Raman bands. It is evident that the intensities of the spectra of the unprinted papers are negligible compared to the printed samples. Thus, the resonance Raman bands of the printed sample seem to originate primarily from the DY86 colorant. The selected laser wavelength thereby appears to enhance the chromophore bands even when the ink is printed onto the paper. The baselines of the spectra are somewhat tilted, because the remaining fluorescence has not been removed by baseline subtraction.

Preliminary tests of the Kerr gate rejection system were also accomplished for coatings printed with other types of ink jet colorants, namely a magenta modified direct dye DV107, and a yellow pigmented ink PY83 (Table 2). The coating containing SA latex-starch polymer system and kaolin as a sole pigment (S0:100) was selected as a substrate. Both of these colorants and the kaolin pigment have proved to cause fluorescence problems in earlier Raman experiments. ${ }^{7,9}$ Figure 2 presents the resonance Raman spectra of the magenta DV107 colorant in solution (Table 2), and as printed on the S0:100 coating before and after light exposure. The spectrum of an unprinted coating S0:100 is shown for control. Figure 3 presents the corresponding spectra for the yellow pigmented ink PY83, when coating S0:100 is used. As previously, additional numerical fluorescence removal procedures were not performed for the spectra, so the curves presented in Figure 2 and Figure 3 represent the "raw data" obtained from the measurement. It is evident that the Kerr gate fluorescence rejection functions effectively in both cases, for the resonance Raman bands of the sample stand clearly out. Furthermore, the Raman bands of the printed samples again appear to originate mainly from the colorants, so the selected excitation wavelength seems to be appropriate for the studies of the chemical paper-ink interactions. Furthermore, as a result of light exposure, the bands of dye-based magenta ink DV107 decrease clearly in intensity, whereas the bands of the pigmented PY83 ink remain relatively unchanged. This is an expected result, because the light fastness of the pigmented ink generally surpasses that of dye-based inks. ${ }^{4}$ It should be noted that in the case of DV107 the laser power had to be slightly lowered because of the sensitivity of the printed samples to degradation. The other measurement conditions were kept unchanged. Despite this, and the sample spinning during the measurement, the printed samples tended to burn somewhat. Consequently, it is possible that there is some incontrollable variation in the results rendering absolute Raman intensities to be less reliably 
observed. However, rather than detecting absolute overall Raman intensities the technique is suited to detecting relative intensity changes within Raman spectra that can result, for example, from the appearance or disappearance of species or conformers in the sample.

Light fastness of ink jet prints on coated papers. Light fastness of ink jet prints on the experimental coatings was studied using the DY86 DEG ink (Tables 1 and 2). Light fastness values based on the CIEL*a*b* color difference $\Delta \mathrm{E}^{*}$ between the unexposed and lightexposed prints are presented in Table 1 . The $\Delta \mathrm{E}^{*}$ data suggest that in the case of PVA-polyDADMAC coatings, light fastness on the coating containing solely kaolin (P0:100) is better than on coatings containing PCC as well. Otherwise, systematic correlations between the PCC:kaolin ratio of the coating and the light fastness values can not be found. On weakly cationic SA latex-starch coatings, on the other hand, light fastness of the studied model ink on kaolin-rich coatings is lower than on PCC-rich coatings. The resonance Raman measurements on the printed samples were accomplished using Kerr gate rejection with 470 $\mathrm{nm}$ excitation wavelength. Examples representing the typical behavior observed for all the studied PVA-poly-DADMAC and latex-starch coatings are presented in Figures 4 and 5, respectively.

In Figures 4 and 5, the uppermost spectrum (a) represents the resonance Raman spectrum of the DY86 colorant in the ink solution, the spectrum (b) corresponds to an unexposed print, and the lowermost spectrum (c) stands for the light-exposed sample. In both cases, certain wavenumber shifts and differences in the band intensities can be observed, if the spectra of the DY86 colorant in liquid state and the unexposed print are examined. This would suggest the presence of chemical interactions between the colorant and the coated paper. This may also result from the difference between liquid phase and solid state (or printed state). The following band assigments should be considered only tentative, and need to be verified using model compounds. First of all, the band at $1623 \mathrm{~cm}^{-1}$ likely representing the aromatic $\mathrm{C}=\mathrm{C}$ stretching ${ }^{25-28}$ shifts to lower wavenumbers, ca. to $1616-1617 \mathrm{~cm}^{-1}$. This is common behavior to both PVA-poly-DADMAC and SA latex-starch coatings. Secondly, the intensity ratio of the bands at $1448 \mathrm{~cm}^{-1}$ and $1396 \mathrm{~cm}^{-1}$ changes as a result of printing. This manifests itself as a slight decrease in the intensity of the $1396 \mathrm{~cm}^{-1}$ band, which possibly represents the symmetric $\mathrm{N}=\mathrm{N}$ stretching ${ }^{25-28}$, relative to the $1448 \mathrm{~cm}^{-1}$ band. On PVA-poly-DADMAC coatings, the band ratio in the spectra of unexposed printed samples is rather independent of the PCC:kaolin ratio, whereas on latex-starch coatings, the ratio change is the most pronounced on kaolin-rich coatings. Added to this, the band at $1232 \mathrm{~cm}^{-1}$ likely originating from the sulphonate group of the DY86 colorant $^{25-28}$ shifts to the higher wavenumber the larger is the proportion of the PCC is in the coating. 
If the resonance Raman spectra measured from the light-exposed prints (spectra c) are considered, the intensity of the azo band ca. at $1393 \mathrm{~cm}^{-1}$ is found to further reduce in relation to the band at $1446 \mathrm{~cm}^{-1}$. This is typical behavior for all the studied DY86 prints, and is more pronounced with the latex-starch coatings (Figure 5) than with PVA-poly-DADMAC coatings (Figure 4). The literature suggests that photo-oxidation of azo dyes is affected by the azohydrazone tautomeric equilibrium, the latter being more prone to the oxidative fading. ${ }^{29-32}$. An earlier study ${ }^{33}$ gave indications that on cationic PVA-poly-DADMAC coatings, the pigment ratio of the coating did not seem to influence on the attachment of the anionic DY86 colorant to the coating, possibly owing to the strong interactions between the colorant and polyDADMAC. Meanwhile, the opposite was found to hold true for the studied weakly cationic SA latex-starch model coatings. Therefore, it would seem reasonable to assume here that the variation in the chemical environment of the colorant would have an influence on its chemical stability, and thereby on light fastness. Consequently, it might be possible that the chemical state of the azo group, which is the chromophore of the DY86 colorant, would differ depending on the pigment composition of the weakly cationic SA latex-starch coatings and thus have an influence on the sensitivity of the colorant to light fading.

The spectra were analyzed further using a multivariate PCA method. The PCA loading plots presented in Figures 6 and 7 represent the resonance Raman spectra of ink jet prints on PVApoly-DADMAC and latex-starch coatings, respectively. The plots depict the difference between the Kerr gated resonance Raman spectra measured from unexposed and lightexposed prints such that the positive contributions ("bands") represent the unexposed prints, whereas the negative ones stand for the light-exposed prints. The fact that some of the positive contributions are located below the $\mathrm{x}$-axis and vice versa, can presumably be explained with the baseline fluctuations that were not removed from the raw data. Besides the azo band at $1395 \mathrm{~cm}^{-1}$, the majority of the other positive contributions in the PCA loading plots represent the DY86 colorant as well. ${ }^{25-28}$ In contrast, the negative contributions at 1072 $\mathrm{cm}^{-1}$ and $647 \mathrm{~cm}^{-1}$ can be assigned to the PCC pigment ${ }^{26-28}$ and the Kerr medium $\left(\mathrm{CS}_{2}\right)$, respectively. It is evident that in the positive side of the loading plots, the magnitude of the the azo band at $1395 \mathrm{~cm}^{-1}$ in relation to the band at $1434 \mathrm{~cm}^{-1}$ is larger for the weakly cationic SA latex-starch coatings (Figure 7) than in the case of PVA-poly-DADMAC coatings. Consequently, this would suggest that the DY86 colorant, particularly the azo bond, photodecomposes and the bands originating from the coating thus become more dominant. This is consistent with the literature. ${ }^{29-31}$ In addition to this, the PCA plots reveal that there is a strong positive contribution at $1434 \mathrm{~cm}^{-1}$ instead of a band at $1445 \mathrm{~cm}^{-1}$ present in the spectra of the printed samples, and a small negative contribution at $1473 \mathrm{~cm}^{-1}$. This might 
indicate that the chemical group causing the Raman band at $1434 \mathrm{~cm}^{-1}$ would take part in the photo-degradation reaction of the colorant, whereas the contribution at $1473 \mathrm{~cm}^{-1}$ for one might originate from some photo-degradation reaction product of the DY86 colorant.

The exact assignment of the resonance Raman bands of the DY86 azo colorant and clarification of its exact degradation and stabilization mechanisms requires more detailed studies. Nevertheless, these preliminary measurements illustrate that resonance Raman spectroscopy combined with the Kerr gate fluorescence rejection system has a great potential both in analyses of paper-ink interactions and light fastness studies of ink jet prints.

\section{CONCLUSIONS}

The purpose of this study was to evaluate the applicability of Kerr gated resonance Raman spectroscopy to the light fastness studies of ink jet prints. This was investigated using model inks and coatings of known compositions.

The results illustrate that resonance Raman spectroscopy equipped with the Kerr gate fluorescence rejection method is well suited to photo-degradation studies of ink jet prints, owing to the effective damping of the sample-related fluorescence, and the ability to obtain resonance enhancement of the desired chemical groups of a colorant. The first feature makes studies of even highly fluorescent samples possible whereas the latter enables investigations of specific chromophore structures. Sample degradation by the pulsed laser does not restrict the method. Furthermore, the method is capable of detecting light-induced changes in the chemical structure of the sample. This would enable elaborate studies of chemical paper-ink interactions, which generally play a decisive role in light fastness of an ink jet prints. The results also give indications that after the light exposure of the ink jet prints, the amount of remaining azo bonds of the DY86 colorant would be smaller in the case of latex coatings than in the case of PVA-poly-DADMAC coatings.

\section{ACKNOWLEDGMENTS}

Financial support from the European Union TMR Large Scale Facility Access Programme, the National Technology Agency of Finland (Tekes), M-Real, Stora Enso, Metso Paper, and the International Ph.D. Programme in the Pulp and Paper Science and Technology (PaPSaT) is gratefully acknowledged. 


\section{REFERENCES}

1. D. Glittenberg, A. Voigt, Paper Tech. 42 (2001) 24.

2. D. I. Lunde, Pulp Paper 73 (1999) 41.

3. B. Thompson, Printing materials: Science and technology, Pira International, United Kingdom, 1998.

4. D. E. Bugner, in A. S. Diamond, D. S. Weiss, Handbook of imaging materials, Marcel Dekker, New York, 2002, p. 603.

5. H. P. Le, J. Imag. Sci. Technol. 42 (1998) 49.

6. C. Rodger, G. Dent, J. M. Watkinson, W. E. Smith, Appl. Spectrosc. 54 (2000) 1567.

7. K. Vikman, K. Sipi, J. Imag. Sci. Technol. 47 (2003) 139.

8. K. Vikman, J. Imag. Sci. Technol. 47 (2003) 30.

9. K. Vikman, T. Vuorinen, Manuscript submitted to Nord. Pulp. Pap. Res. J.

10. C. L. Stevenson, T. Vo-Dinh, in J. J. Laserna, Modern Techniques In Raman Spectroscopy, John Wiley \& Sons, Chichester, 1996, p. 22.

11. C. H. Munro, W. E. Smith, P. C. White, Analyst 120 (1995) 993.

12. J. Vyörykkä, M. Halttunen, H. Iitti, J. Tenhunen, T. Vuorinen, P. Stenius, Appl. Spectrosc. 56 (2002) 776.

13. P. P. Yaney, J. Opt. Soc. Am. 62 (1972) 1297.

14. J. M. Harris, R. W. Chrisman, F. E. Lytle, R. S. Tobias, Anal. Chem. 48 (1976) 1937.

15. T. Tahara, H. Hamaguchi, Appl. Spectrosc. 47 (1993) 391.

16. J. Howard, N. J. Everall, R. W. Jackson, K. Hutchinson, J. Phys. E: Sci. Instrum. 19 (1986) 934.

17. T. Fujii, K. Kamogawa, T. Kitagawa, Chem. Phys. Lett. 148 (1988) 17.

18. P. Matousek, M. Towrie, A. Stanley, A. W. Parker, Appl. Spectrosc. 53 (1999) 1485.

19. P. Matousek, M. Towrie, C. Ma, W. M. Kwok, D. Phillips, W. T. Toner, A. W. Parker, J. Raman Spectrosc. 32 (2001) 983. 
20. P. Matousek, M. Towrie, A. W. Parker, J. Raman Spectrosc. 33 (2002) 238.

21. T. Fujino, T. Tahara, J. Phys. Chem. A. 104 (2000) 4203.

22. N. Everall, T. Hahn, P. Matousek, A. W. Parker, M. Towrie, Appl. Spectrosc. 55 (2001) 1701.

23. A.-M. Saariaho, A.-S. Jääskeläinen, P. Matousek, M. Towrie, A. W. Parker, T. Vuorinen, Holtzforschung 58 (2004) 1.

24. L. Eriksson, E. Johansson, N. Kettaneh-Wold, S. Wold, Multi- and Megavariate Data Analysis. Principles and Applications, Umetrics Academy, Sweden, 2001.

25. P. J. Trotter, Appl. Spectrosc. 31 (1977) 30.

26. F. R. Dollish, W. G. Fateley, F. F. Bentley, Characteristic Raman Frequencies of Organic Molecules, John Wiley \& Sons, New York, London, 1974.

27. N. B. Colthup, L. H. Daly, S. E. Wiberley, Introduction to Infrared and Raman Spectroscopy, Academic Press, San Diego, 1990.

28. D. Lin-Vien, N. B. Colthup, W. G. Fateley, J. G. Grasselli, The Handbook of Infrared and Raman Characteristic Frequencies of Organic Molecules, Academic Press, San Diego, 1991.

29. J. Griffiths, C. Hawkins, J. Chem. Soc. Chem. Comm. (1972) 463.

30. J. Griffiths, C. Hawkins, J. Chem. Soc. Perkin II (1977) 747.

31. N. Kuramoto, in A. T. Peters, H. S. Freeman, Physico-Chemical Principles of Color Chemistry (Advances in Color Chemistry Series, Vol. 4), Blackie Academic \& Professional, London, 1996, p. 196.

32. W. J. Wnek, M. A. Andreottola, P. F. Doll, S. M. Kelly, in A. S. Diamond, D. S. Weiss, Handbook of imaging materials, Marcel Dekker, New York, 2002, p. 531.

33. K. Vikman, T. Vuorinen, J. Imag. Sci. Technol. 48 (2004) 138. 
Table 1. The chemical compositions of the model coatings and light fastness values of ink jet prints printed with DY86 DEG ink after $100 \mathrm{~h}$ light exposure.

\begin{tabular}{|c|c|c|c|c|c|}
\hline \multirow{2}{*}{$\begin{array}{l}\text { Coating } \\
\text { Code }\end{array}$} & \multicolumn{2}{|l|}{ Pigment } & \multirow[t]{2}{*}{ Binder } & \multirow[t]{2}{*}{ Dispersant } & \multirow[t]{2}{*}{$\Delta \mathrm{E}^{*}$} \\
\hline & PCC, pph & Kaolin, pph & & & \\
\hline P0:100 & 0 & 100 & PVA 10 pph & Poly-DADMAC & 17.8 \\
\hline P20:80 & 20 & 80 & & Ј рріп & 25.6 \\
\hline P40:60 & 40 & 60 & & & 24.0 \\
\hline P60:40 & 60 & 40 & & & 22.4 \\
\hline P80:20 & 80 & 20 & & & 21.3 \\
\hline P100:0 & 100 & 0 & & & 26.6 \\
\hline S0:100 & 0 & 100 & Weakly cationic & Weakly cationic & 27.8 \\
\hline S20:80 & 20 & 80 & & & 26.1 \\
\hline S40:60 & 40 & 60 & & & 17.5 \\
\hline S60:40 & 60 & 40 & & & 18.3 \\
\hline S80:20 & 80 & 20 & & & 19.7 \\
\hline S100:0 & 100 & 0 & & & 20.4 \\
\hline
\end{tabular}

Table 2. Compositions of the model inks.

\begin{tabular}{|l|l|l|l|l|}
\hline \multirow{2}{*}{ Ink Code } & Colorant Type & \multicolumn{2}{|l|}{ Organic Co-Solvent } & \multirow{2}{*}{ Distilled Water, wt. \% } \\
\cline { 3 - 4 } & & Type & Amount, wt. \% & \\
\hline DY86 DEG & DY 86 & Diethylene glycol & 10 & 86 \\
\hline DV107 & DV 107 & 2-pyrrolidone & 20 & 76 \\
\hline PY83 & PY 83 & $\begin{array}{l}\text { Polyethylene } \\
\text { glycol }\end{array}$ & 15 & - \\
\hline
\end{tabular}




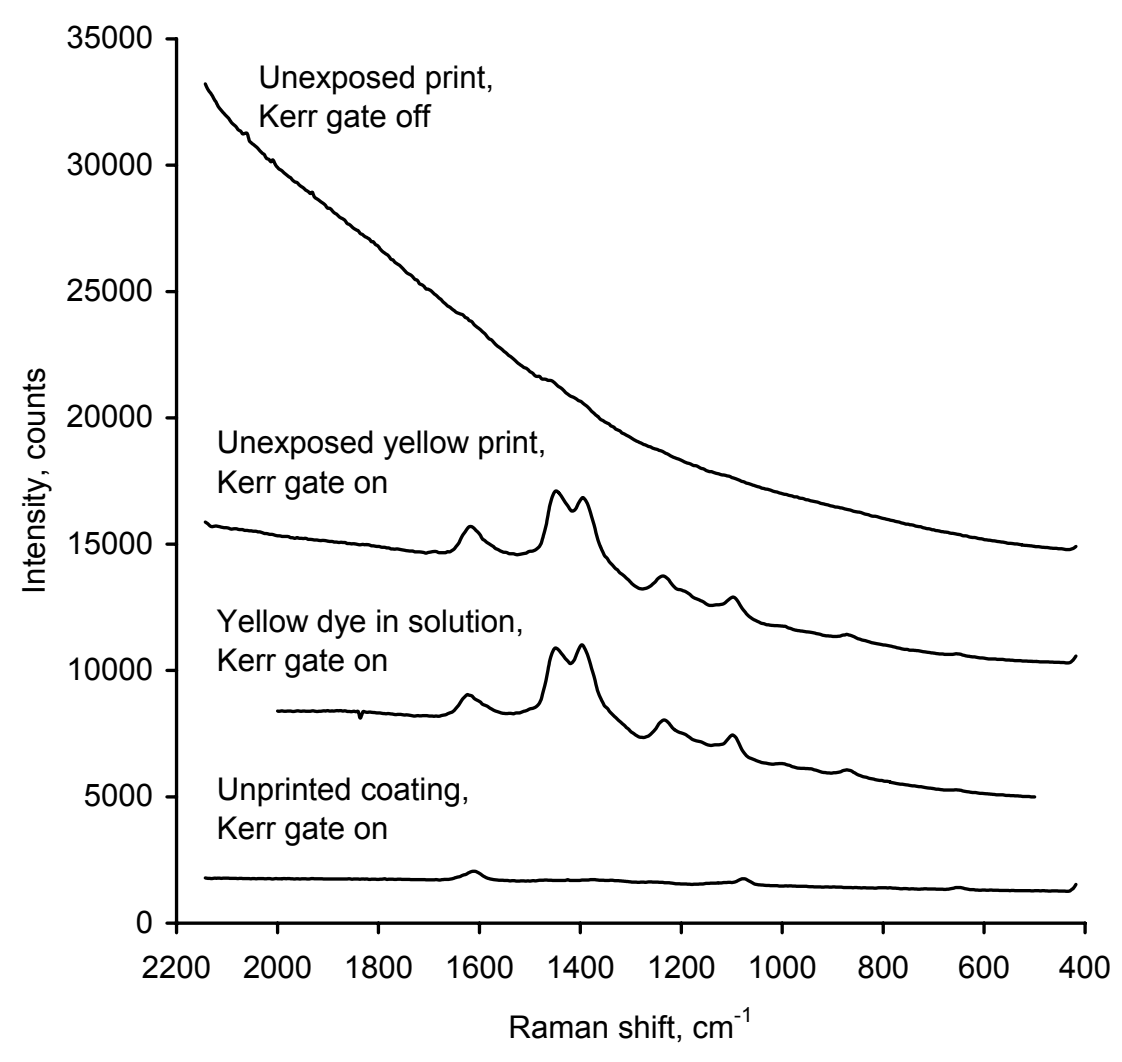

Figure 1. Effect of usage of Kerr gate on resonance Raman spectra of ink jet prints. Colorant DY86, coating P80:20. The spectra are vertically offset for clarity and scaled to fit into the same figure. 


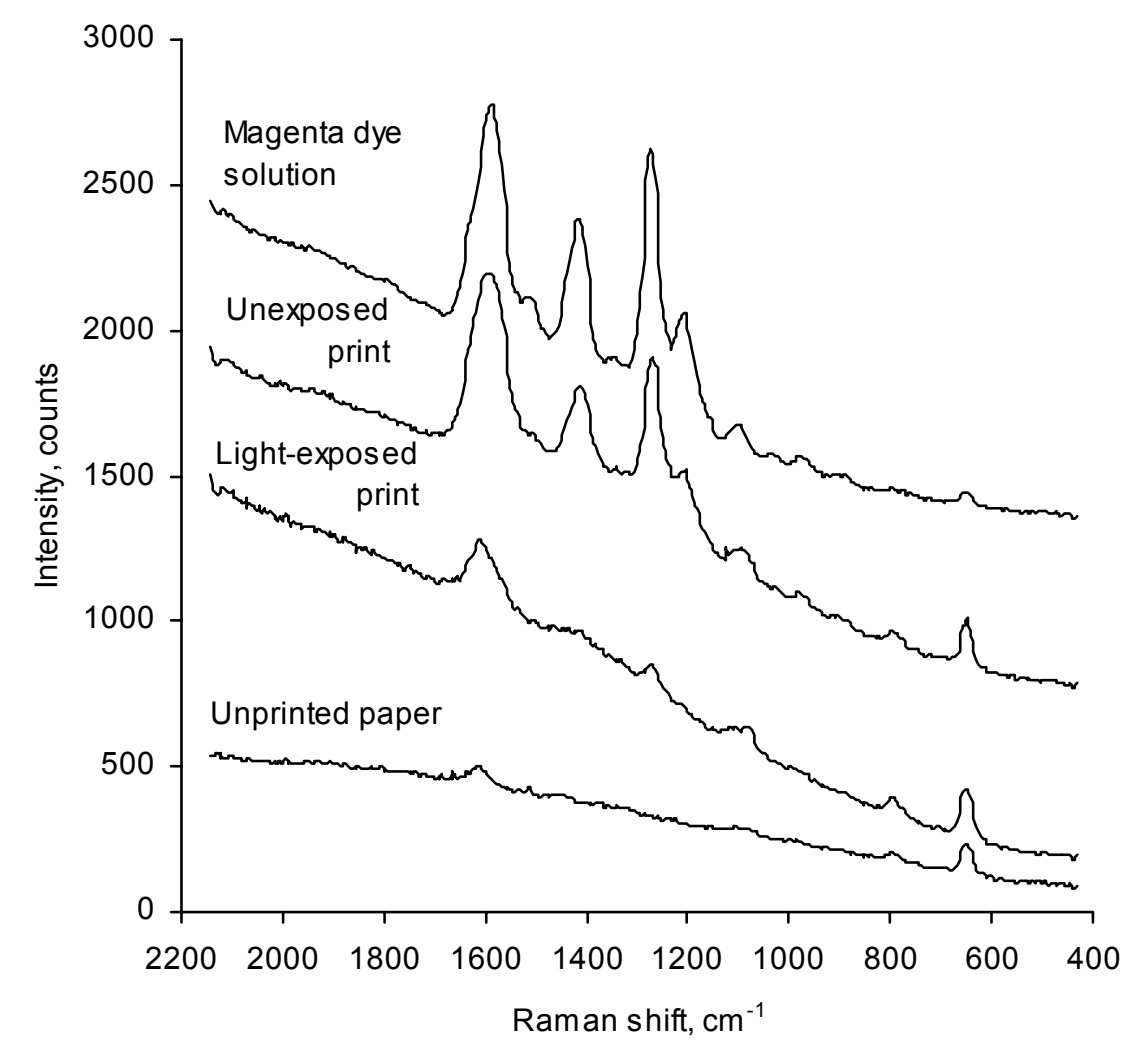

Figure 2. Kerr gated resonance Raman spectra of liquid magenta ink, and corresponding ink jet prints with different light exposure conditions. Colorant DV107, coating S0:100. The spectra are vertically offset for clarity. 


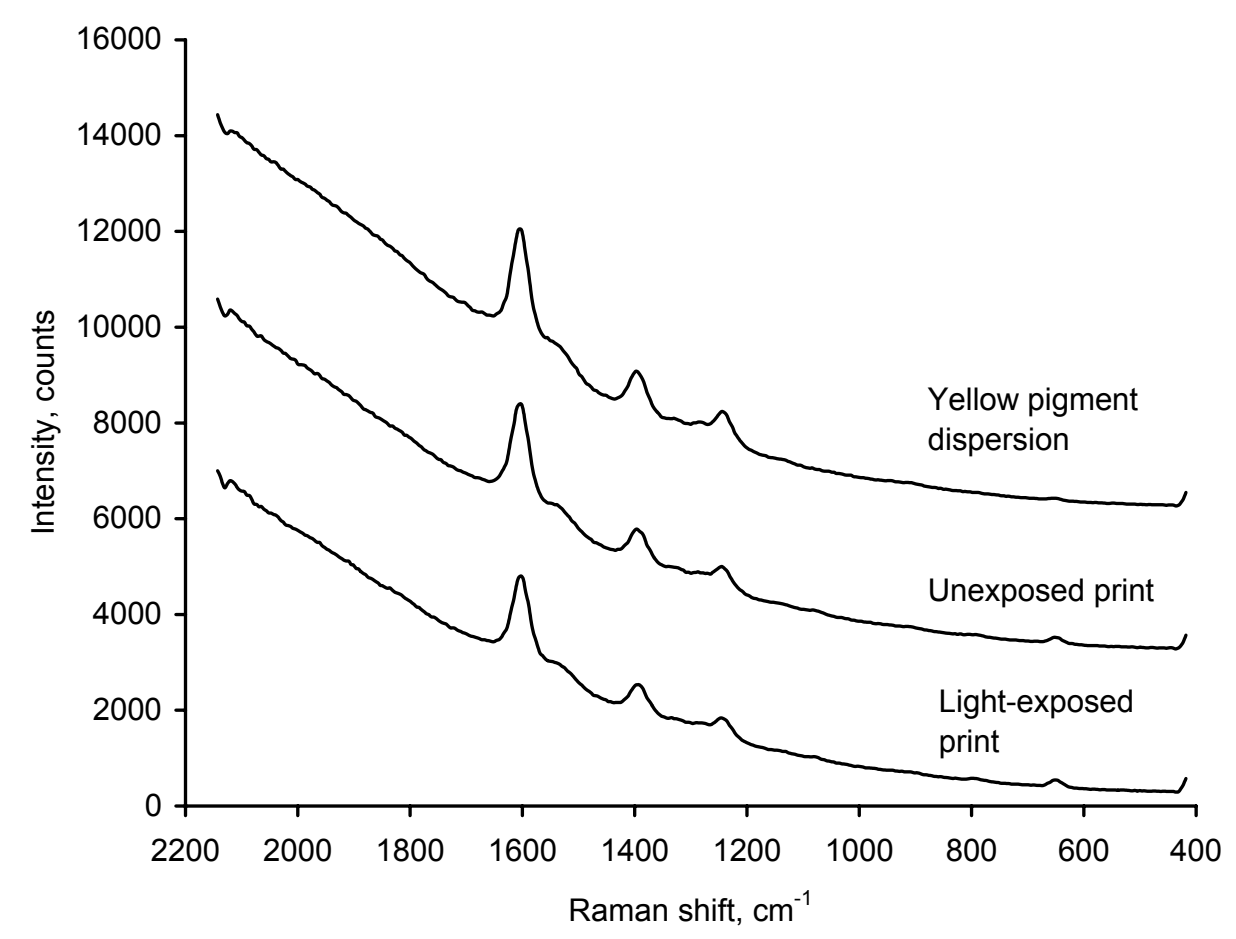

Figure 3. Kerr gated resonance Raman spectra of pigmented yellow ink, and corresponding ink jet prints with different light exposure conditions. Colorant PY83, coating S0:100. The spectra are vertically offset for clarity. 


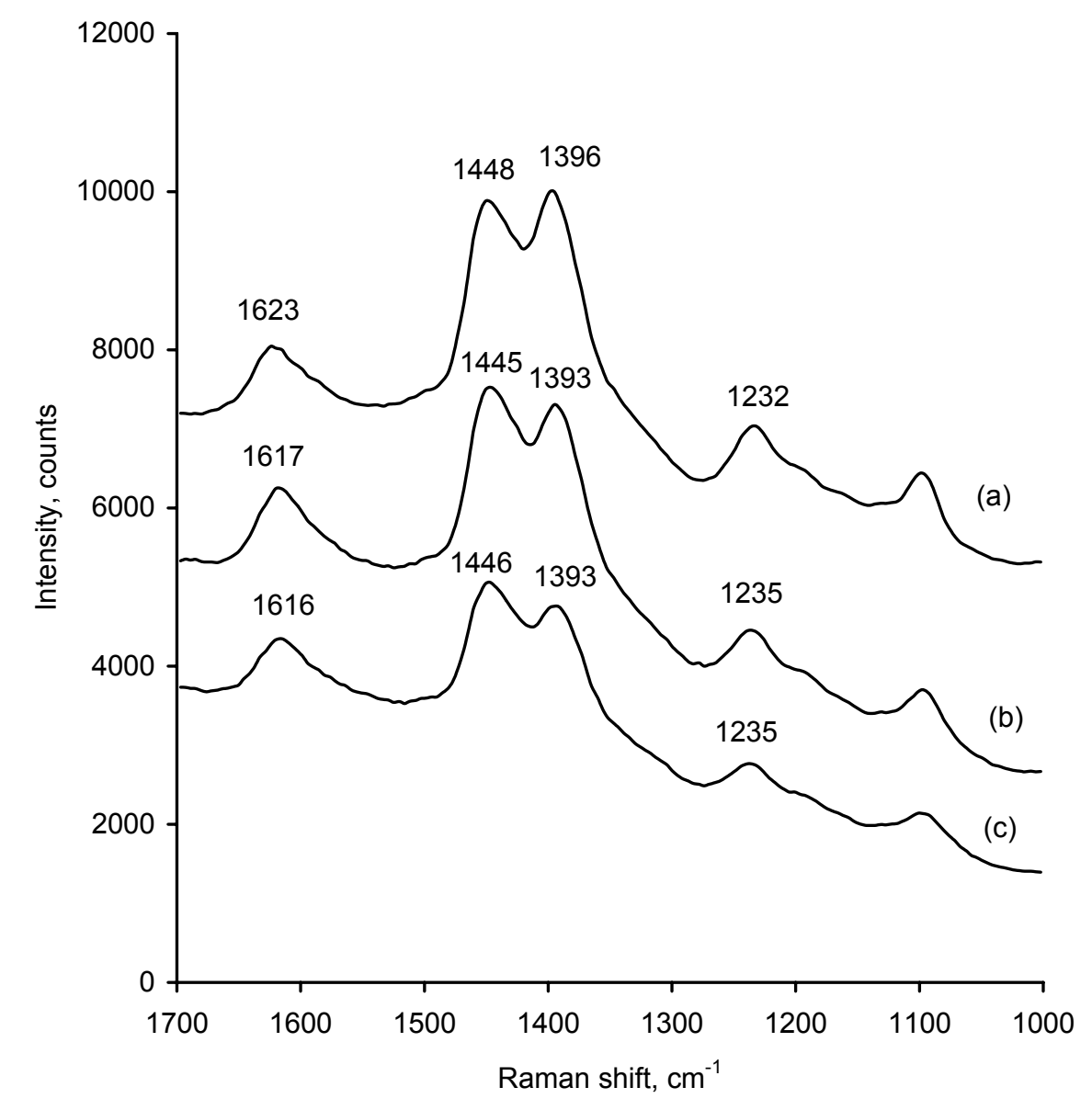

Figure 4. Resonance Raman spectra of (a) DY86 DEG ink in liquid state, (b) unexposed ink jet print, and (c) light-exposed ink jet prints on PVA-poly-DADMAC coating (P60:40). The spectra are vertically offset for clarity. 


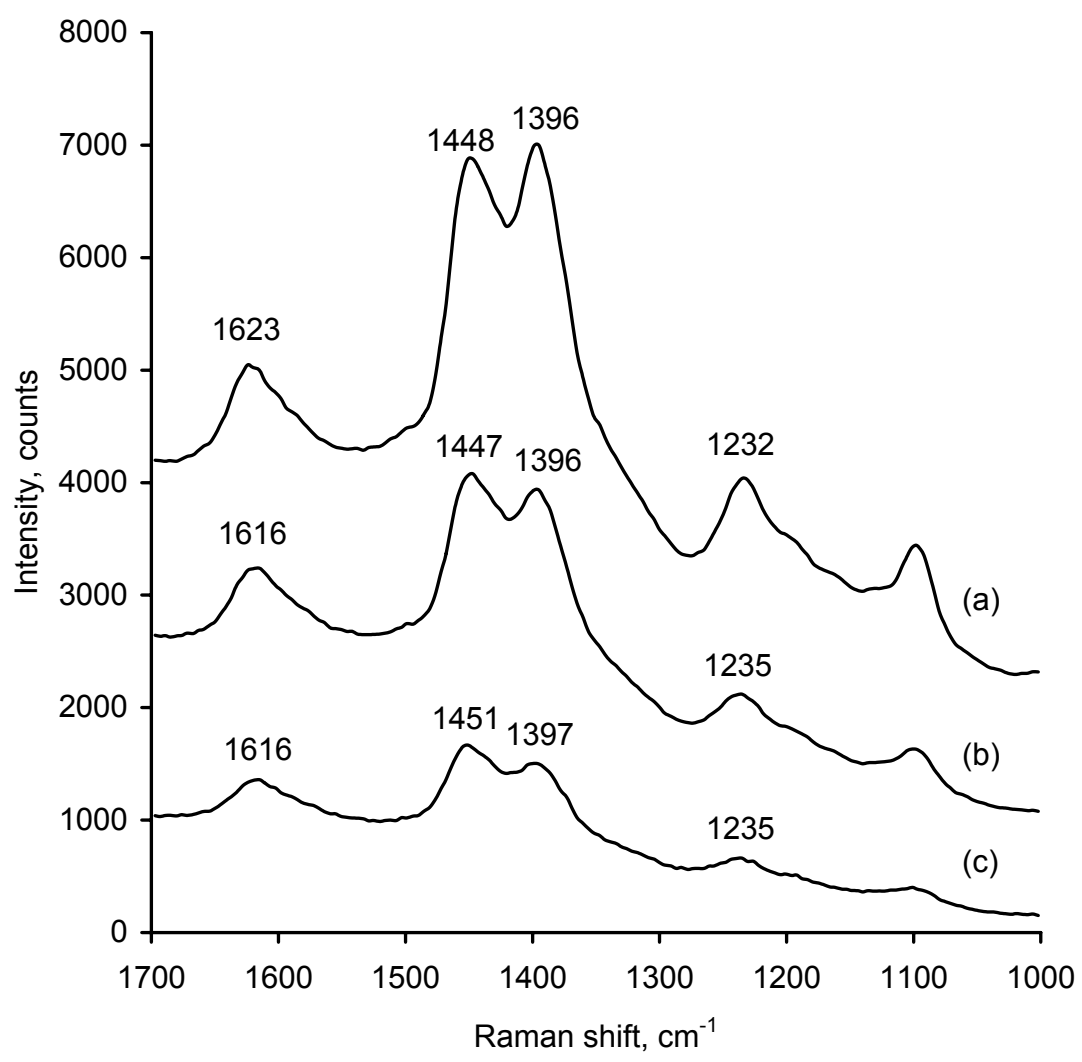

Figure 5. Resonance Raman spectra of (a) DY86 DEG ink in liquid state, and (b) unexposed and (c) light-exposed ink jet prints on weakly cationic SA latex-starch coating (S0:100). The spectra are vertically offset for clarity. 


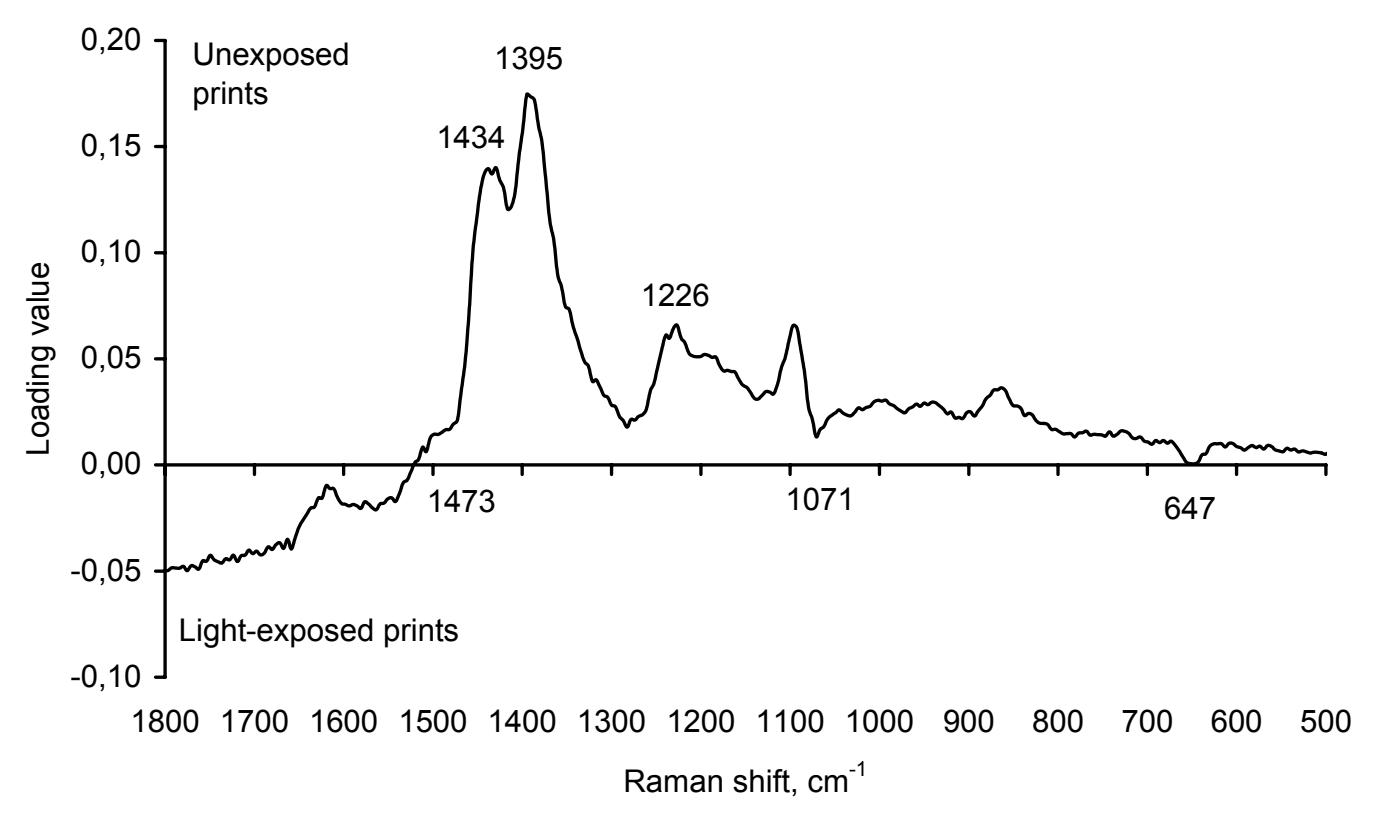

Figure 6. The PCA loading values of untreated and light-exposed prints on PVA-polyDADMAC coatings.

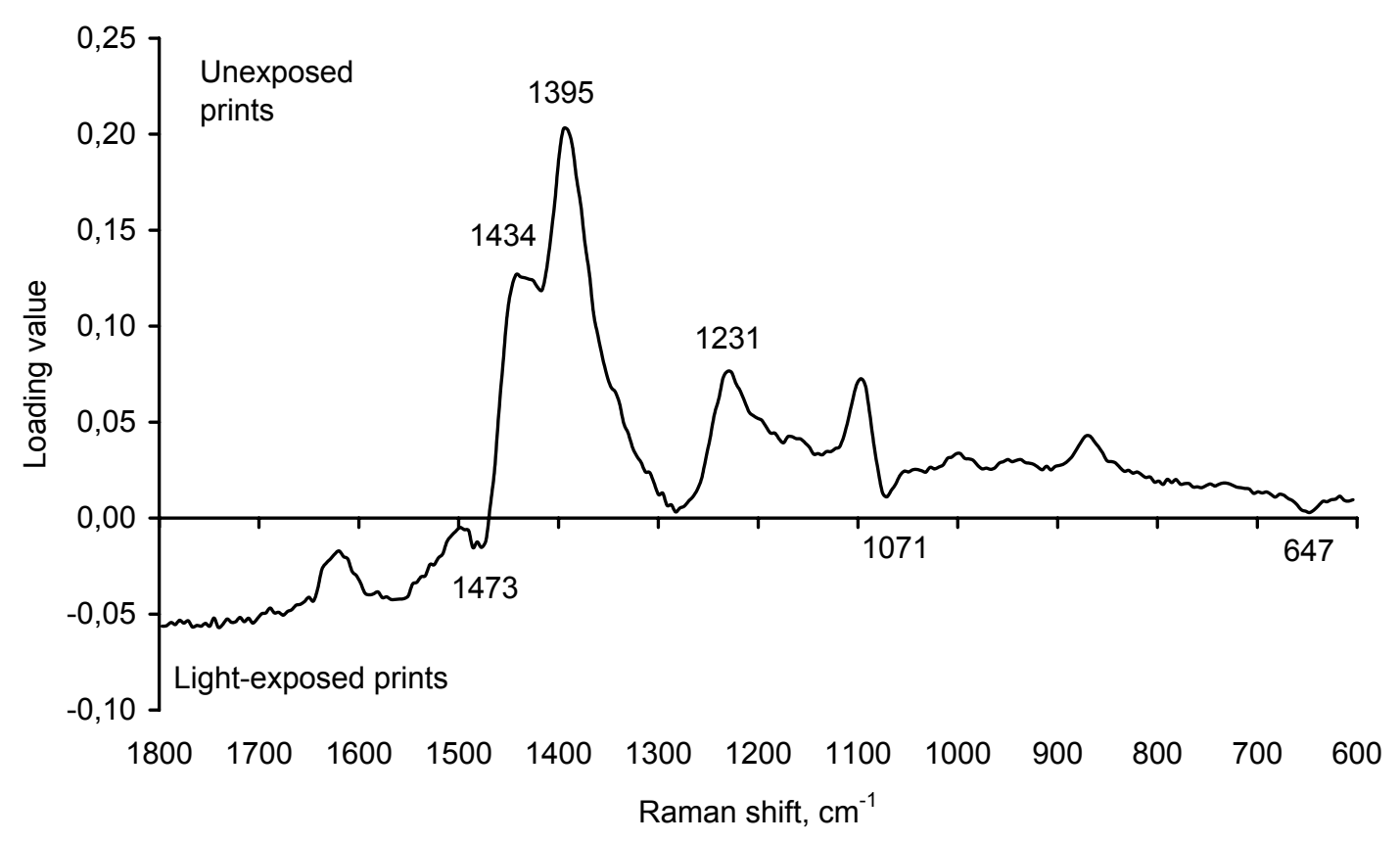

Figure 7. The PCA loading values of untreated and light-exposed prints on weakly cationic SA latex-starch coatings. 